\title{
Patterned emission of organic light emitting diodes with laser irradiation
}

\author{
Ryota Sugimoto, Masahiro Morimoto, and Shigeki Naka \\ Graduate School of Science and Engineering, University of Toyama, \\ 3190 Gofuku, Toyama, 930-8555 Japan, nak@eng.u-toyama.ac.jp
}

\begin{abstract}
We have researched on patterned emission of organic light-emitting diodes. The organic layer was deposited followed by laser patterning in atmosphere. The laser irradiation part was quenched, and it was shown that arbitrary patterning was possible. This technique is expected as a fabrication technique for an on-demand pattern emission.
\end{abstract}

\section{Introduction}

Organic light-emitting diodes (OLEDs) have attracted much interest, such as self-emission, high brightness, high contrast, and so on. They have practically used in the various applications such as a lighting and a display for smartphones and televisions. For various device applications, a variety of simple patterning techniques are required. However, this is difficult with conventional mask patterning. This is because the cost of mask preparation could be large for various requirements, deterioration of the mask, and the like. Free patterning is required without a photomasks or shadow masks.

In this research, we have studied of patterned emission in OLEDs using photo-oxidation $[1,2]$ of the organic semiconductors without the conventional mask. The patterned OLEDs were evaluated the characteristics of emission.

\section{Experimental Methods}

The indium-tin-oxide (ITO) substrates were cleaned by UV-ozone treatment. N,N'-di-1-naphthyl-N,N'diphenylbenzidine ( $\alpha$-NPD) was used as a hole transport layer $(70 \mathrm{~nm}), 5,6,11,12$-tetraphenyltetracene (rubrene) was used as a light emitting layer $(1 \mathrm{~nm})$ and bis(8hydroxy-2-methylquinoline)-(4-phenylphenoxy)

aluminium (BAlq) was used as an electron transport layer $(30 \mathrm{~nm})$. All layers were formed on the ITO substrate by vacuum evaporation. Subsequently, bluelight laser (wavelength of $445 \mathrm{~nm}$ ) was irradiated from

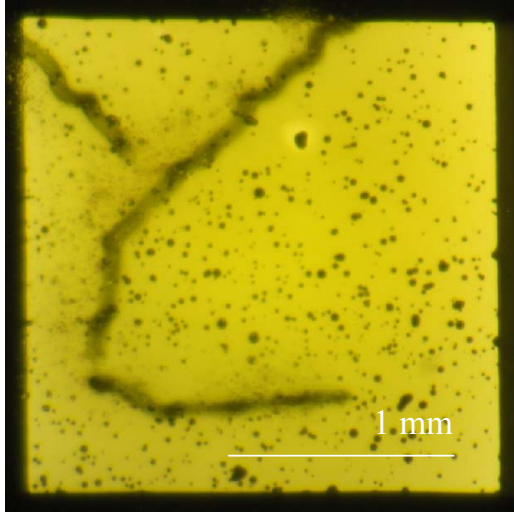

Fig. 2. Patterned emission image in hiragana character of "to".

formed as an electrode. The blue-light for light patterning was used FABOOL Laser Mini (SmartDIY) and controled the scan speed to be $500-8,000 \mathrm{~mm} / \mathrm{min}$ and the output power to be $0.08-1.6 \mathrm{~W}$. The FABOOL Laser Mini is freely manipulated in the $\mathrm{X}$ and $\mathrm{Y}$ axes.

\section{Results and Discussions}

The patterned devices were fabricated under the various laser conditions. The patterning of the hiragana character of "to" was controlled with an output of $0.08 \mathrm{~W}$ and the number of six times irradiation. The light emission photograph of patterning is shown in Fig. 2. A clear patterning could be achieved by reducing the scanning speed. However, the pattern position and the pattern of (a)

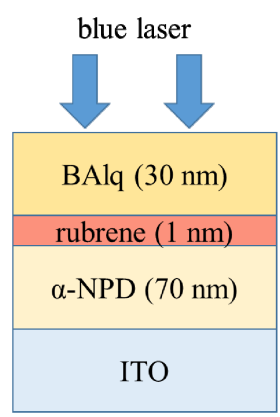

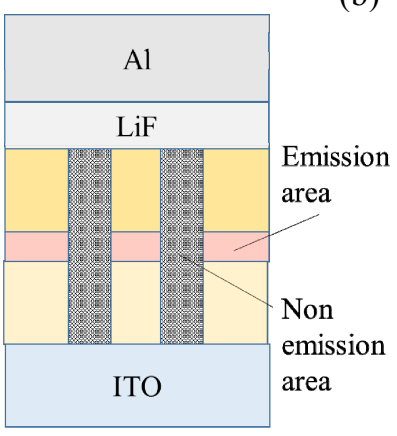

(b)

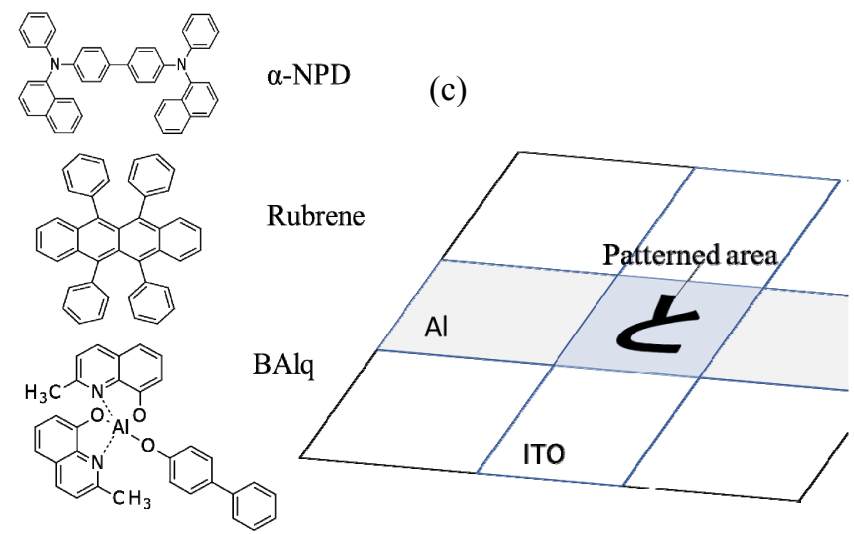

Fig. 1. (a) Device structure of before and after patterning. (b) Chemical structures of organic materials. (c) Schematic image of patterning. 
(a)

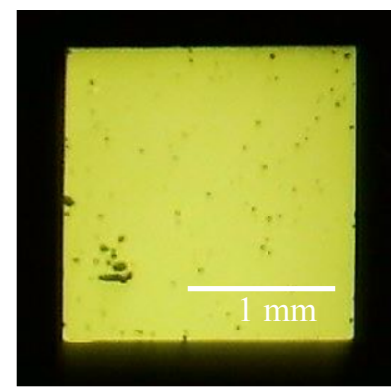

(c)

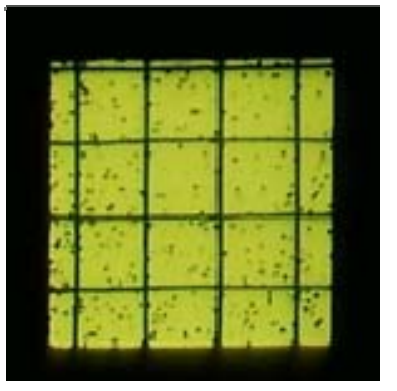

Fig. 3. Emission image of (a) pristine device and laser patterned device with scanning speed with (b) 8,000 $\mathrm{mm} / \mathrm{min}$ (c) $5,000 \mathrm{~mm} / \mathrm{min}$ (d) $1,000 \mathrm{~mm} / \mathrm{min}$.

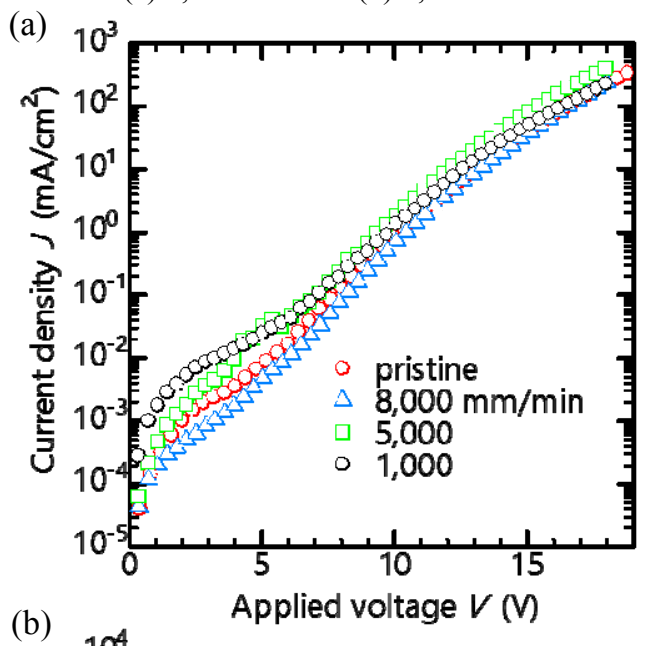

(b)

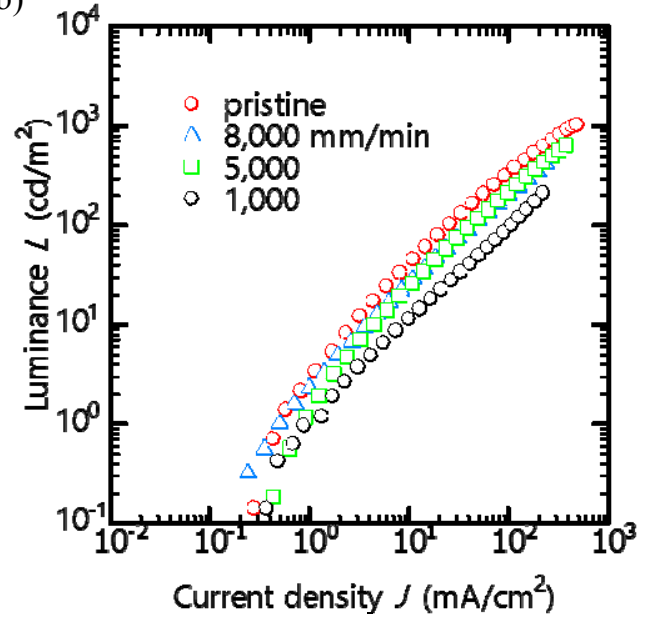

the curve part were disordered. The blue-light was irradiated at patterning with a maximum output for simple controlling because the output of the blue-light was controlled by the duty ratio. The scanning speed was changed at maximum output to control patterning.

The light emitting area was patterned by patterning the entire substrate. Fig. 3 shows the emission photograph at $100 \%$ output and scanning speed of 1,000 , 5,000 and $8,000 \mathrm{~mm} / \mathrm{min}$. The light patterning in a grid was successful. Fig. 4 shows the characteristics of the grid patterned devices. The current density of the devices according to the scanning speed of the laser was 0.82 , $0.71,717,13.3 \mathrm{~mA} / \mathrm{cm}^{2}$ at $10 \mathrm{~V}$, and the luminance characteristics were $2.14,1.58,3.12,1.18 \mathrm{~cd} / \mathrm{m}^{2}$. No leak current was observed even in the low voltage region of 2 $\mathrm{V}$ or less. Depending on the irradiation rate, the deterioration and damage of the device characteristics were confirmed. The same characteristics as obtained without laser irradiation were obtained under the condition of suitable scanning speed. The pattern became clearer and thicker with the scanning speed decreasing. Past experiments confirmed patterning even for devices without rubrene. This pattern is probably not due to photo-oxidation but due to a direct pattern by laser. However, no major deterioration of the device was fabricated, and it can be used as a wide variety of ondemand patterning.

\section{Conclusions}

Pattern emission of OLED by laser irradiation was studied. Though the degradation of the device characteristics was confirmed depending on the irradiation quantity of the laser, under the condition of the appropriate laser irradiation quantity, the characteristics equivalent to no laser irradiation were obtained. This pattern may be due to direct laser patterning rather than photo-oxidation. However, no significant degradation of the device was observed. This method can be used as a wide range of on-demand patterning methods.

\section{Acknowledgements}

This work was supported by Kenjiro Takayanagi Foundation and JSPS KAKENHI Grant Numbers JP19K04465.

\section{References}

[1] S. Shimizu, A. Uchida, S. Oshima, I. Onishi, and S. Fujisawa, "Photo-oxidation of rubrene", Crystallographic Society of Japan, 37, 33 (1995) [in Japanese].

[2] S. Shirai and J. Kido, "Fabrication using of Multi Color Polymer EL Devices the Photo-bleaching Method", J Photopolym. Sci. Technol, 14, 317-322 (2001).

Fig. 4 (a) Current density-voltage ( $J-V)$ and, (b) Luminancecurrent density $(L-J)$, of OLEDs according to the scanning speed. 